\title{
HIGHLIGHTS
}

\section{Satraplatin delays progression in prostate cancer}

Prostate cancer is the second most common cancer in men worldwide. The FDA approved docetaxel in 2004 as first-line treatment for men with castraterefractory prostate cancer (CRPC) and this is now the standard treatment for such patients. Patients with metastatic disease, however, eventually discontinue docetaxel treatment because of disease progression or toxic effects. It is therefore important to identify effective and well-tolerated treatments for patients with CRPC, whose experience disease progression after chemotherapy, as appropriate second-line therapy is an important unmet medical need.

Satraplatin is a novel oral platinum compound that has demonstrated preclinical activity in prostate cancer cell lines that are resistant to cisplatin, taxanes and anthracyclines. In a phase II trial, satraplatin was shown to have activity in CRPC patients, and a randomized phase III trial of satraplatin plus predisone versus predisone alone demonstrated a significant progression-free survival advantage for the satraplatin-treated patients. A multinational, randomized, placebo-controlled, phase III trial was initiated to determine the efficacy and tolerability of satraplatin in men with metastatic CRPC who had previously had disease progression after chemotherapy. In total, 950 patients were randomly assigned to receive $80 \mathrm{mg} / \mathrm{m} 2$ satraplatin orally once daily and $5 \mathrm{mg}$ predisone twice daily or the same prednisone regimen alone.

There was a $33 \%$ reduction in the risk of progression or death with satraplatin compared with placebo (hazard ratio $0.67 ; 95 \% \mathrm{CI} 0.57-0.77)$. The overall survival was similar in the two treatment arms. Compared with the placebo arm, satraplatin significantly reduced the time to pain progression (hazard ratio 0.64; 95\% CI 0.51-0.79). Satraplatin was generally well tolerated, although hematologic toxic effects, such as myelosuppression, and gastrointestinal disorders were more frequent with satraplatin. These results suggset activity in patients with CRPC who experience progression after initial chemotherapy.

The researchers conclude that "Orally administered satraplatin was well tolerated in patients with advanced CRPC, with beneficial effects on disease progression and pain. Satraplatin chemotherapy may potentially address an unmet need in patients with CRPC whose disease progressed after initial chemotherapy". The authors plan to evaluate the utility of biomarkers that could be useful in identifying the subgroup(s) of patients that are most likely to benefit from satraplatin.

\section{Lisa Hutchinson}

Original article Sternberg, C. N. et al. Multinational, double-blind, phase III study of prednisone and either satraplatin or placebo in patients with castrate-refractory prostate cancer progressing after prior chemotherapy: The SPARC Trial. J. Clin. Oncol. 27, 5431-5438 (2009) 